\title{
FREE VIBRATION ANALYSIS OF THIN CYLINDRICAL SHELLS SUBJECTED TO INTERNAL PRESSURE AND FINITE ELEMENT
}

\author{
ANALYSIS \\ J. Kandasamy ${ }^{1}$, M. Madhavi², N. Haritha ${ }^{3}$ \\ ${ }^{1}$ Corresponding author Department of Mechanical Engineering, M.V.S.R Engineering College, Nadergul-501 510 \\ professorkandan@gmail.com \\ ${ }^{2}$ Department of Mechanical Engineering, M.V.S.R Engineering College, Nadergul - 501510 \\ madhavimatlapudi72@gmail.com \\ ${ }^{3}$ Department of Mechanical Engineering, M.V.S.R Engineering College, Nadergul - 501510 \\ nukaraju.haritha7@gmail.com
}

\begin{abstract}
The paper presents the dynamic characteristics of thin cylindrical shells subjected to unpressurized and pressurized conditions. Emphasis is given to develop theoretical models based on LOVE's first approximations and Donnell's assumptions to the higher order linear differential equations of thin cylindrical shell governing equations to determine Eigen values and Eigen Vectors of the shells subjected to uniform internal pressure. The characteristic equation thus obtained from model is a cubic equation and results in three roots for the three dimensional shell. Axial, circumferential and radial frequencies and mode shapes are generated for different pressurized conditions. The variation of radial frequency at higher values of circumferential nodes is predominant as compared to axial and circumferential frequencies. Numerical techniques for pressurized conditions are computed to compare with the theoretical model developed.
\end{abstract}

Keywords: Free Vibration, Thin Cylindrical Shells, Internal Pressure, Mathematical Modeling, Fea

\section{INTRODUCTION}

Pressure vessel is a closed container designed to hold gases or liquids at a pressure substantially different from the ambient pressure. So far pressure vessels under static conditions are discussed, when a pressure vessel is considered to be as a moving body then it is subjected to dynamic or time-independent loading which will force the shell to a vibratory motion from its position of static equilibrium. The resonance effect is caused due to these vibrations. In order to avoid resonance it is necessary to study the dynamic characteristics of cylindrical shells. Cylindrical shells are widely used for commercial under water vehicles, in aerospace applications and in automotive sectors as pressure bottles. During operation these cylindrical shells are subjected to radial, circumferential as well as axial loads, which alter the natural frequency of these structures. To avoid resonance due to fluctuating component the dynamic behavior of the shell structures has to be studied. One such application of pressure vessels for studying the dynamic characteristics includes CNG pressure vessels, made of fully metal, hoop wrapped with metal liner, fully wrapped with metal liner or fully composite.

Pressure vessels are important because many liquids and gases are to be stored under high pressure. Emphasis is placed upon the strength of the vessel to prevent explosions as a result of rupture. Most pressure vessels required to carry only low pressures and thus are constructed of tubes and sheets rolled to form cylinders. Some pressure vessels must carry high pressures, however, and the thickness of the vessel wall must be increased in order to provide adequate strength. The assemblies, containing thin shells, find wide use in modern engineering, especially in ships, aircraft and spacecraft industry. The vibration frequencies of shells are important in engineering where shell structures are commonly used as structural components in engineering design. Basically a shell structure is a three-dimensional structure. The dynamic characteristics of shells have been studied by many researchers. Love employed Kirchhoff hypothesis for shells and formulated classical shell theory (Love, 1927). The natural frequencies of cylindrical shells are clustered in a very narrow band and they are thus prone to becoming involved in resonant vibrations. Thin cylindrical shells are those whose wall thickness is small (less than $1 / 20$ of the diameter of the cylinder) compared to the radius of curvature and the corresponding radius of twist. Cylindrical and spherical pressure vessels are commonly used for storing gas and liquids under pressure. A pressurized shell, the main source of vibrations and noise is to be designed for high strength, low vibration and noise radiation characteristics. Thus, frequencies and mode shapes of such structures are important in the design of systems. Traditionally the behavior of shells is studied under static loading conditions. A shell may also be subjected to dynamic or time-dependent loading which will force the shell to a vibratory motion from its position of static equilibrium. Such a motion is referred to as forced vibration. 
On the other hand, if a shell vibrates without any external loading it is called free vibration (Ramachandran, 1993). The destructive effect of resonance with nearby oscillating or rotating equipment may be avoided if the fundamental frequencies of the structures are known beforehand.

Previous investigations on the vibration of thin-walled cylinders seem to be limited mostly to the unpressurized case. The free vibrations of thin cylinders in the case of negligible bending stiffness have been considered by Rayleigh (Timoshenko, 1940). The solution is relatively simple inclusion of the bending stiffness of the walls of the shells make problem much more complicated. Rayleigh derived an expression for the frequencies of thin cylinders in which the motion of all cross sections was identical. This corresponds to the fundamental axial form for a free ended cylinder. The general equations of flexural vibration of the walls of cylinders were later investigated by Love but no frequency equations for any specific end conditions were given. The frequency equation for a cylinder with freely supported ends was first proposed by Flugge (Flugge, 1934). For freely supported ends, frequency equations based on strain energy relations by Timoshenko were experimentally verified with considerable accuracy (Arnold and Warburton, 1953). The natural frequency of thin walls may actually decrease as the number of circumferential nodes increases due to the proportion of strain energy. The vibration of pressurized cylinder has been discussed by Stern under the hypotheses that the skin vibrates normal to the static position and that the stresses in the vibration modes are effectively equal to the stresses generated by the internal pressure Later Serb has solved the problem for the lowest frequencies for nearly- inextensible vibration modes by Rayleigh method. Rayleigh showed that a great simplification in the analysis of shell vibrations can be achieved if tangential inertia forces can be neglected. It is then necessary to consider only one component of displacement-the transverse, or radial component. Reissner shows that the simplified equations as "shallow shell" theory that gives an accurate description of the transverse vibration of cylinders provided that the number of circumferential waves, $\mathrm{n}$, is sufficiently large (Reissner, 1955). Reissner developed theoretical models and applied to thin walled circular cylinders subjected to internal pressure in which numbers of circumferential modes are relatively small and by considering different pressure values. The frequency spectra and vibration modes obtained from theoretical models are compared with numerical techniques (Reissner, 1955).

The basic assumptions of Love's first approximation are used. To account for the effect of internal pressure, the interaction of the membrane stresses and the change of curvature are included in the equations of equilibrium. By considering the Hamilton's principle the governing equations for the vibration of shell is determined. By applying the Donell's assumptions to the obtained governing equations and by considering the axisymmetric case, natural frequencies of thin cylindrical shells under free vibrations are determined.

\section{MATHEMATICAL MODELING OF THIN CYLINDRICAL SHELLS FOR DYNAMIC CHARACTERISTICS}

A set of simplifying assumptions that provide a reasonable description of the behavior of thin elastic shells is used to derive the equilibrium equations that are consistent with the assumed displacement field.

The assumptions, known as LOVE's first approximations are

(a) the thickness $\mathrm{h}$ is quite small when compared with other dimensions and also with its radii of curvature.

(b) the elastic displacements are small in comparison with the thickness of the shell.

(c) the stress normal to the middle surface is negligible.

(d) The normal to the middle surface before deformation remains normal even after deformation.

The curvilinear coordinate system shown in Fig.1. and Fig.2 represents a thin cylinder with freely supported ends. The $\mathrm{x}$ axis is directed along the generator of the cylinder, $y=a \theta$ is measured clockwise in the circumferential direction, and the $\mathrm{z}$-axis is directed inward along the positive normal to the middle surface of the shell. Let $\mathrm{u}, \mathrm{v}, \mathrm{w}$ be the components of the displacement of a point on the middle surface of the shell in the $\mathrm{x}, \mathrm{y}$ and $\mathrm{z}$ directions respectively. The governing equations of vibration of shells are obtained starting from the Hamilton's principle.

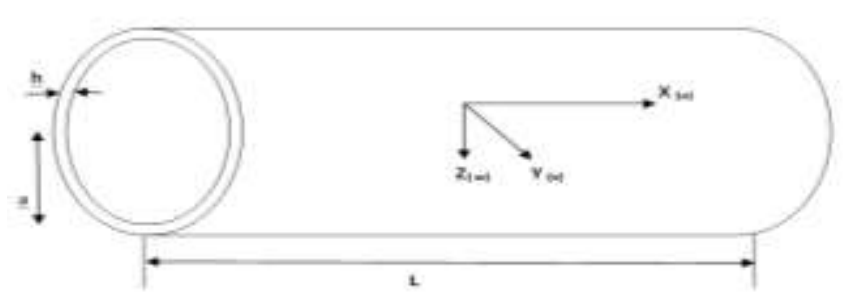

Fig.1. Coordinate system

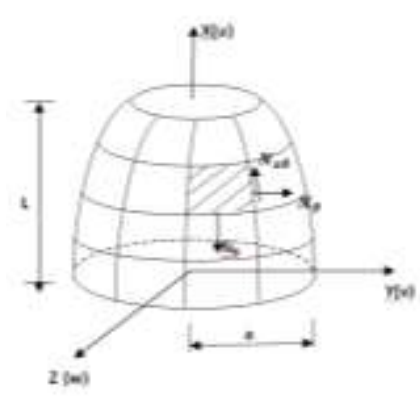

Fig.2. Stress resultants

The Hamilton's principle states that among the set of all admissible configurations of a system, the actual motion makes the quantity $\int_{t_{1}}^{t_{1}} L d t$ stationary, provided the configuration is known at the limits $t=t_{1}$ and $t=t_{2}$. According to Hamilton's principle 
$\delta \int_{t_{1}}^{T_{2}} L d t=\delta \int_{t_{1}}^{T_{2}}[T-(U-V)] d t=0$

where $\mathrm{T}$ is kinetic energy, $\mathrm{U}$ is strain energy and $\mathrm{V}$ is work potential of all applied loads. The governing differential equations, the strain energy due to loads, kinetic energy and formulations of the general dynamic problem are derived on the basis of Hamilton's principle. The equation of motion is obtained by taking a differential element of shell with internal forces like membrane $\left(\mathrm{N}_{1}, \mathrm{~N}_{2}\right.$ and $\left.\mathrm{N}_{6}\right)$ shearing forces $\left(Q_{1}\right.$ and $\left.Q_{2}\right)$ and the moment resultants $\left(M_{1}, M_{2}\right.$ and $\mathrm{M}_{6}$ ).

\section{EQUATION OF EQUILIBRIUM}

By applying the dynamic version of the Hamilton's principle and substituting the parameters of strain energy and the kinetic energy as given in equation (3.1) and integrating the displacement gradients by parts, the resulting equation is

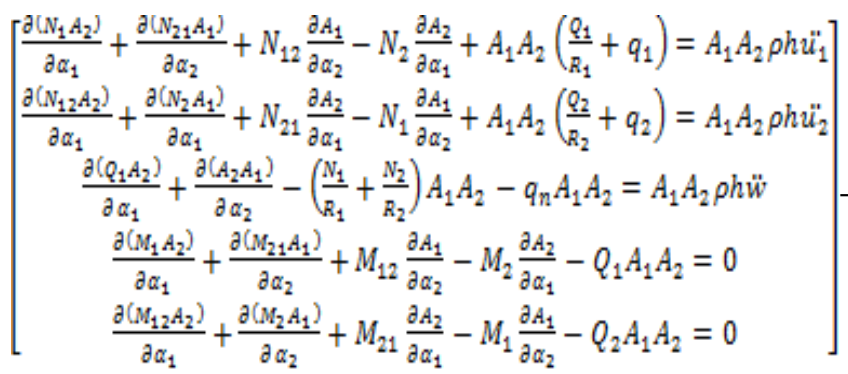
(3.2)

The governing equations derived in orthogonal curvilinear coordinates for general shell element is reduced for circular cylindrical shell. The equations of motion are given as

$$
\begin{aligned}
& {\left[\begin{array}{c}
\frac{\partial N_{x}}{\partial x}+\frac{\partial N_{s x}}{a \partial s}-\frac{\overline{N_{s}}}{a}\left(\frac{\partial^{2} w}{\partial x \partial s}-\frac{\partial w}{\partial x}\right)-\rho h \frac{\partial^{2} u}{\partial t^{2}}=0 \\
\frac{\partial N_{x s}}{\partial x}+\frac{\partial N_{x}}{a \partial s}-\frac{Q_{x}}{a}+\overline{N_{x}} \frac{\partial^{2} v}{\partial x^{2}}-\rho h \frac{\partial^{2} v}{\partial t^{2}}=0 \\
\frac{\partial Q_{x}}{\partial x}+\frac{\partial Q_{x}}{a \partial s}+\frac{N_{s}}{a}+\overline{N_{x}} \frac{\partial^{2} W}{\partial x^{2}}+\frac{\overline{N_{s}}}{a}\left(\frac{\partial v}{a \partial s}+\frac{\partial^{2} w}{a \partial s^{2}}\right)-\rho h \frac{\partial^{2} W}{\partial t^{2}}=0
\end{array}\right]} \\
& {\left[\begin{array}{l}
\frac{\partial M_{x}}{\partial x}+\frac{\partial M_{s x}}{a \partial s}-Q_{x}=0 \\
-\frac{\partial M_{x x}}{\partial x}+\frac{\partial M_{s}}{a \partial s}-Q_{s}=0
\end{array}\right]}
\end{aligned}
$$

\section{UNPRESSURIZED CYLINDRICAL SHELLS}

According to Donnell's assumptions,

(a) Neglecting the effect of transverse shear force, while considering equilibrium of forces in circumferential direction

(b) The effect of stretching displacement may be neglected while considering curvature displacement relations.

Frequency parameter, $\varphi=\frac{\rho\left(1-\theta^{2}\right) \omega^{2} a^{2}}{E}=\left(1-\vartheta^{2}\right) x$
Membrane stress, $n_{\theta}=\frac{\overline{N_{\alpha}}\left(1-\theta^{2}\right)}{E h}=\left(1-\vartheta^{2}\right) \overline{n_{g}}$

$n_{x}=\frac{\overline{N_{x}}\left(1-\theta^{2}\right)}{E h}=\left(1-\vartheta^{2}\right) \overline{n_{x}}$

Axial wavelength factor, $\lambda=\frac{m \pi a}{L}$

Shell parameter, $\beta=\frac{\hbar^{2}}{12 a^{2}}$

The following three equations are obtained

$$
\begin{aligned}
& {\left[\left(-\lambda^{2}-\frac{1-\mu}{2} m^{2}+\varphi\right) \nu+\left(\frac{1+p}{2} n-\lambda n n_{\varphi}\right) V+\left(-\mu \lambda+\lambda m_{q}\right) V=0\right.} \\
& \left.\frac{1+\mu}{2} \ln \nabla+\left(-\frac{1-\mu}{2} x^{2}-n^{2}-\beta\left[n^{2}+(1-\mu) x^{2}\right]-n_{3}\right)^{2}+\phi\right)\left(\gamma+\left(n+\beta n^{2}+\beta \lambda^{2} n\right) W=0\right.
\end{aligned}
$$

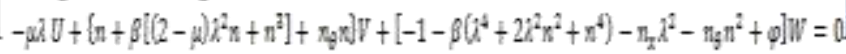

For a non trivial solution the determinant of the coefficients of $\mathrm{U}, \mathrm{V}, \mathrm{W}$ in above equations must vanish resulting to the following equation

$$
\varphi^{\mathrm{a}}-K_{2}^{\prime} \varphi^{2}+K_{1}^{\prime} \varphi-K_{0}^{\prime}=0
$$

The characteristic equation (3.11) thus obtained from model is a cubic equation and results in three roots for three dimensional shell. The three roots results in Eigen Values and Eigen Vectors in axial, circumferential and radial directions. Frequencies and mode shapes are generated for both pressurized and unpressurised conditions.

\section{PRESSURIZED CYLINDRICAL SHELLS:}

In the case of pressurized thin cylindrical shells the frequency equation is similar to that of unpressurized cylindrical shells except that the membrane stresses are replaced with the pressure terms as

$\overline{N_{x}}=\frac{P a}{2}, \overline{N_{\theta}}=P a$

then the characteristic equation becomes

$\varphi^{3}-K^{v}{ }_{2} \varphi^{2}+K^{s}{ }_{1} \varphi-K^{s}{ }_{0}=0$

Where reverting to the notations $\overline{N_{x_{1}}} \overline{N_{\theta}}$.

Here $\overline{n_{x}}=\frac{\overline{N_{x_{x}}}}{E h_{h}}=\frac{P_{a}}{E h_{h}}, \overline{n_{\theta}}=\frac{\overline{\bar{N}_{\theta_{s}}}}{E h_{h}}=\frac{P a}{2 E h}$

$\left[\begin{array}{c}K_{0}^{\prime}=K_{0}+a_{1} \overline{n_{\theta}}+a_{2} \overline{n_{x}}+a_{2} \overline{n_{\theta}} \overline{n_{x}}+a_{4}{\overline{n_{x}}}^{2}+a_{5}{\overline{n_{\theta}}}^{2} \\ K_{1}^{\prime}=K_{1}+b_{1} \overline{n_{\theta}}+b_{2} \overline{n_{x}}+x^{2} \lambda^{2} \overline{n_{\theta}} \overline{n_{x}}+\lambda^{4}{\overline{n_{x}}}^{2} \\ K_{2}^{\prime}=K_{2}+n^{2} \overline{n_{\theta}}+2 \lambda^{2} \overline{n_{x}}\end{array}\right]$

When the internal pressure vanishes, the coefficients $K_{0}^{v}{ }_{0} K_{1}^{v} K^{v}{ }_{2}$ reduce to $K_{0}, K_{1}, K_{2}$.

The expressions are: 


$$
\begin{aligned}
& \text { [ } K_{0}=\frac{1}{2}\left(1-\mu^{2}\right)(1+\mu) \lambda^{4}+\frac{1}{2}(1-\mu) \beta\left[\left(\lambda^{2}+n^{2}\right)^{4}-2\left(4-\mu^{2}\right) \lambda^{4} n^{2}-8 \lambda^{2} n^{4}-2 n^{6}+4\left(1-\mu^{2}\right) \lambda^{4}+4 \lambda^{2} n^{2}+n^{4}\right] \\
& K_{2}=\frac{3}{2}(1-\mu)\left(\lambda^{2}+n^{2}\right)^{2}+\frac{3}{2}\left(3-\mu-2 \mu^{2}\right) \lambda^{2}+\frac{1}{2}(1-\mu) n^{2}+\beta\left[\frac{1}{2}(3-\mu)\left(\lambda^{2}+n^{2}\right)^{2}+2(1-\mu) \lambda^{4}-\left(2-\mu^{2}\right) \lambda^{2} n^{2}-\frac{1}{2}(3+\mu) n^{4}+2(1-\mu) \lambda^{2}+n^{2}\right. \\
& K_{2}=1+\frac{1}{2}\left(\lambda^{2}+n^{2}\right)(3-\mu)+\beta\left[\left(\lambda^{2}+n^{2}\right)^{2}+2(1-\mu) \lambda^{2}+n^{2}\right.
\end{aligned}
$$

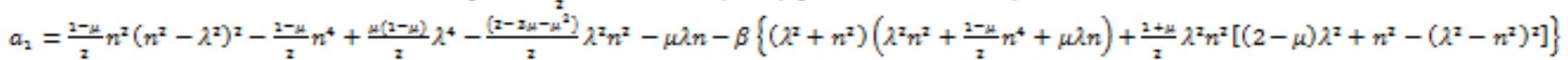

$$
\begin{aligned}
& a_{2}=\lambda^{2}\left[\left(1-\mu^{2}\right) \lambda^{2}+\frac{\lambda-w}{x}\left[n^{2}+\left(n^{2}-\lambda^{2}\right)^{2}\right]+\beta\left(\lambda^{2}+n^{2}\right)^{2}\left(\lambda^{2}+\frac{\lambda-w}{z} m^{2}\right)\right. \\
& a_{x}=\lambda^{2}\left[\frac{x-w}{z} \lambda^{2} n^{2}+\frac{2-w}{z} n^{4}+\mu \lambda^{2}\right] \\
& a_{4}=\lambda^{4}\left(\lambda^{2}+\frac{\lambda^{2}-w_{1}}{x} n^{2}\right) \\
& a_{s}=\frac{2+4 x^{2}}{2} \lambda^{2} n^{2}\left(n^{2}-1\right) \\
& b_{1}=\frac{\pi-\mu}{z} n^{4}+2 \lambda^{2} n^{2}-n^{2}+\mu \lambda^{2}-\beta n^{2}\left(\lambda^{2}+n^{2}\right) \\
& b_{2}=\frac{2-\underline{x}}{x} \lambda^{4}+\frac{3-z_{\mu}}{x} \lambda^{2} n^{2}+\lambda^{2}+\beta \lambda^{2}\left(\lambda^{2}+n^{2}\right)^{2}
\end{aligned}
$$

3.14

The equation so obtained is used in calculating the natural frequencies of cylindrical shells subjected to pressurized conditions. The theoretical model is programmed in MATLAB and validated with numerical technique using ANSYS software (Nitin, 2008) for the axial, circumferential and radial natural frequencies along with their respective mode shapes. In the present study, a CNG pressure vessel made of AISI grade $18 \mathrm{Ni}$ (200) Maraging steel (annealed) material, subjected to internal pressure ranging between 0 to 200 for varied radius to thickness and length to radius ratios. The material properties are as follows: Young's modulus, $E=183 \mathrm{GPa}$, Poisson's ratio $\mu=0.3$, Density $\rho=7990 \mathrm{~kg} / \mathrm{m}^{3}$. Dimensions of the cylinder: Length of the cylinder, $\mathrm{L}=706$ $\mathrm{mm}$, Radius of the cylinder, $\mathrm{a}=170 \mathrm{~mm}$, Thickness of cylinder, $\mathrm{h}=\mathrm{t}=7.89 \mathrm{~mm}$, axial wave length factor is calculated as $\lambda=0.756$ and for circumferential nodes, $n=1$, $2,34,5,6,7,8,9,10$. Axial nodes, $m=1$ the frequencies are calculated respectively. The results obtained from the theoretical model from MATLAB codes are tabulated in Table. 1. to Table. 13. The model is generated in ANSYS and similar boundary conditions are applied. The results obtained for a Pressure of $\mathrm{P}=5$ bar and at nodal value $\mathrm{n}=5$ is shown in Figure 3 to Fig. 8 and compared with the theoretical results in Table.14. The frequency variation along the axial, circumferential and radial directions for different $\mathrm{a} / \mathrm{h}$ ratios and for varying number of lobes are shown in Figure. 9 to 14 respectively.

Theoretical Results: Unpressurized condition for different values of $\mathbf{a} / \mathbf{h}$ ratio

Table.1. $\mu=0.3, \mathrm{a} / \mathrm{h}=170 / 7.89=21.54, \lambda=0.756, \Delta \mathrm{P}=0 \mathrm{bar}$

\begin{tabular}{|c|c|c|c|c|c|c|c|c|c|c|}
\hline Number of lobes $n$ & $\mathrm{n}=1$ & $\mathrm{n}=2$ & $\mathrm{n}=3$ & $\mathrm{n}=4$ & $\mathrm{n}=5$ & $n=6$ & $\mathrm{n}=7$ & $\mathrm{n}=8$ & $\mathrm{n}=9$ & $\mathrm{n}=10$ \\
\hline Axial frequency $\mathrm{f}_{1}, \mathrm{~Hz}$ & 304.225 & 400.77 & 522.92 & 655.41 & 793.21 & 934.34 & 1077.5 & 1221.6 & 1366.6 & 1512.2 \\
\hline $\begin{array}{c}\text { Circumferential frequency } \mathrm{f}_{2}, \\
\mathrm{~Hz}\end{array}$ & 172.62 & 245.52 & 316.48 & 392.21 & 471.99 & 549.7 & 633.4 & 718.9 & 804.89 & 891.2 \\
\hline Radial frequency $\mathrm{f}_{3}, \mathrm{~Hz}$ & 115.8 & 71.22 & 52.50 & 59.77 & 76.88 & 146.19 & 58.09 & 71.99 & 87.98 & 105.758 \\
\hline
\end{tabular}

\begin{tabular}{|c|c|c|c|c|c|c|c|c|c|c|}
\hline Number of lobes $\mathrm{n}$ & 1 & 2 & 3 & 4 & 5 & 6 & 7 & 8 & 9 & 10 \\
\hline Axial frequency $\mathrm{f}_{1}, \mathrm{~Hz}$ & 226.45 & 346.62 & 481.41 & 621.98 & 765.32 & 910.39 & 1056.3 & 1202.9 & 1350.0 & 1497.2 \\
\hline $\begin{array}{c}\text { Circumferential frequency } \mathrm{f}_{2}, \\
\mathrm{~Hz}\end{array}$ & 121.83 & 193.92 & 274.45 & 358.94 & 445.05 & 531.78 & 618.91 & 706.2 & 793.7 & 881.28 \\
\hline Radial frequency $\mathrm{f}_{3}, \mathrm{~Hz}$ & 52.08 & 70.29 & 61.055 & 96.84 & 147.895 & 69.66 & 95.560 & 125.4 & 159.2 & 197.03 \\
\hline
\end{tabular}

Table.2. $\mu=0.3, \mathrm{a} / \mathrm{h}=370 / 7.89=46.89, \lambda=0.756, \Delta \mathrm{P}=0 \mathrm{bar}$

\begin{tabular}{|c|c|c|c|c|c|c|c|c|c|c|}
\hline Number of lobes $\mathrm{n}$ & $\mathrm{n}=1$ & $\mathrm{n}=2$ & $\mathrm{n}=3$ & $\mathrm{n}=4$ & $\mathrm{n}=5$ & $\mathrm{n}=6$ & $\mathrm{n}=7$ & $\mathrm{n}=8$ & $\mathrm{n}=9$ & $\mathrm{n}=10$ \\
\hline $\begin{array}{c}\text { Axial frequency } \mathrm{f}_{1}, \mathrm{~Hz} \\
\begin{array}{c}\text { Circumferential frequency } \mathrm{f}_{2}, \\
\mathrm{~Hz}\end{array}\end{array}$ & 173.94 & 234.16 & 306.89 & 384.04 & 465.05 & 548.31 & 632.93 & 7148.47 & 804.49 & 890.95 \\
\hline${\text { Radial frequency } \mathrm{f}_{3}, \mathrm{~Hz}}^{\mathrm{H}}$ & 104.80 & 72.454 & 137.16 & 98.62 & 95.329 & 115.13 & 148.29 & 159.96 & 175.29 & 192.51 \\
\hline
\end{tabular}

Pressurized condition for different values of $\mathbf{a} / \mathbf{h}$ ratio

Table.3. $\mu=0.3, \mathrm{a} / \mathrm{h}=170 / 7.89=21.54, \lambda=0.756, \Delta \mathrm{P}=100 \mathrm{bar}$

\begin{tabular}{|c|c|c|c|c|c|c|c|c|c|c|}
\hline Number of lobes $\mathrm{n}$ & $\mathrm{n}=1$ & $\mathrm{n}=2$ & $\mathrm{n}=3$ & $\mathrm{n}=4$ & $\mathrm{n}=5$ & $\mathrm{n}=6$ & $\mathrm{n}=7$ & $\mathrm{n}=8$ & $\mathrm{n}=9$ & $\mathrm{n}=10$ \\
\hline Axial frequency $\mathrm{f}_{1}, \mathrm{~Hz}$ & 227.30 & 347.06 & 481.80 & 623.04 & 767.39 & 910.46 & 1056.4 & 1202.8 & 1350.0 & 1497.3 \\
\hline $\begin{array}{c}\text { Circumferential frequency } \mathrm{f}_{2}, \\
\mathrm{~Hz}\end{array}$ & 125.02 & 196.33 & 273.70 & 359.84 & 447.70 & 531.82 & 618.9 & 706.45 & 793.81 & 881.42 \\
\hline Radial frequency $\mathrm{f}_{3}, \mathrm{~Hz}$ & 54.32 & 92.643 & 93.42 & 123.11 & 156.892 & 72.750 & 98.62 & 127.93 & 163.35 & 207.19 \\
\hline
\end{tabular}

Table.4. $\mu=0.3, \mathrm{a} / \mathrm{h}=370 / 7.89=46.89, \lambda=0.756, \Delta \mathrm{P}=100 \mathrm{bar}$ 
Table.5. $\mu=0.3, \mathrm{a} / \mathrm{h}=170 / 7.89=21.54, \lambda=0.756, \Delta \mathrm{P}=200 \mathrm{bar}$

\begin{tabular}{|c|c|c|c|c|c|c|c|c|c|c|}
\hline Number of lobes $\mathrm{n}$ & $\mathrm{n}=1$ & $\mathrm{n}=2$ & $\mathrm{n}=3$ & $\mathrm{n}=4$ & $\mathrm{n}=5$ & $\mathrm{n}=6$ & $\mathrm{n}=7$ & $\mathrm{n}=8$ & $\mathrm{n}=9$ & $\mathrm{n}=10$ \\
\hline Axial frequency $\mathrm{f}_{1}, \mathrm{~Hz}$ & 228.62 & 348.93 & 482.70 & 624.88 & 768.99 & 910.52 & 1056.4 & 1202.8 & 1351.9 & 1497.63 \\
\hline $\begin{array}{c}\text { Circumferential frequency } \mathrm{f}_{2}, \\
\mathrm{~Hz}\end{array}$ & 126.44 & 197.21 & 274.65 & 360.92 & 448.81 & 531.91 & 618.98 & 706.45 & 793.96 & 881.80 \\
\hline${\text { Radial frequency } \mathrm{f}_{3}, \mathrm{~Hz}}$ & 54.99 & 94.006 & 95.903 & 128.81 & 59.741 & 75.583 & 110.60 & 141.28 & 173.44 & 215.31 \\
\hline
\end{tabular}

Table.6. $\mu=0.3, \mathrm{a} / \mathrm{h}=370 / 7.89=46.89, \lambda=0.756, \lambda=0.756, \Delta \mathrm{P}=200 \mathrm{bar}$

\begin{tabular}{|c|c|c|c|c|c|c|c|c|c|c|c|}
\hline Number of lobes $n$ & $\mathrm{n}=1$ & $\mathrm{n}=$ & $\mathrm{n}=$ & & & $=5$ & $=6$ & $=7$ & $=8$ & $\mathrm{n}=9$ & $\mathrm{n}=10$ \\
\hline \multicolumn{2}{|c|}{ Axial frequency $\mathrm{f}_{1}, \mathrm{~Hz}$} & 286.37 & 399.23 & 521.59 & 654.32 & 792.26 & 934.718 & 1077.5 & 1221.5 & 1366.4 & 1512.1 \\
\hline \multicolumn{2}{|c|}{$\begin{array}{c}\text { Circumferential frequency } \mathrm{f}_{2}, \\
\mathrm{~Hz}\end{array}$} & 203.96 & 256.46 & 326.06 & 400.44 & 479.15 & 549.49 & 633.98 & 719.36 & 805.37 & 891.66 \\
\hline \multicolumn{2}{|c|}{ Radial frequency $\mathrm{f}_{3}, \mathrm{~Hz}$} & 70.76 & 124.52 & 60.154 & 77.883 & 103.53 & 54.163 & 67.408 & 84.64 & 103.66 & 116.22 \\
\hline
\end{tabular}

Theoretical results: Unpressurized condition for different values of $1 / a$ ratio

Table.7. $\mu=0.3,1 / \mathrm{a}=706 / 170=4.15, \lambda=0.756, \Delta \mathrm{P}=0 \mathrm{bar}$

\begin{tabular}{|c|c|c|c|c|c|c|c|c|c|c|}
\hline Number of lobes $\mathrm{n}$ & $\mathrm{n}=1$ & $\mathrm{n}=2$ & $\mathrm{n}=3$ & $\mathrm{n}=4$ & $\mathrm{n}=5$ & $\mathrm{n}=6$ & $\mathrm{n}=7$ & $\mathrm{n}=8$ & $\mathrm{n}=9$ & $\mathrm{n}=10$ \\
\hline Axial frequency $\mathrm{f}_{1}, \mathrm{~Hz}$ & 226.45 & 346.62 & 481.41 & 621.98 & 765.32 & 910.39 & 1056.3 & 1202.9 & 1350.0 & 1497.2 \\
\hline $\begin{array}{c}\text { Circumferential frequency } \mathrm{f}_{2}, \\
\mathrm{~Hz}\end{array}$ & 121.83 & 193.92 & 274.45 & 358.94 & 445.05 & 531.78 & 618.91 & 706.2 & 793.7 & 881.28 \\
\hline Radial frequency $\mathrm{f}_{3}, \mathrm{~Hz}$ & 52.08 & 70.29 & 61.055 & 96.84 & 147.895 & 69.66 & 95.560 & 125.4 & 159.2 & 197.03 \\
\hline
\end{tabular}

Table.8. $\mu=0.3,1 / \mathrm{a}=906 / 170=5.329, \lambda=0.589, \Delta \mathrm{P}=0 \mathrm{bar}$

\begin{tabular}{|c|c|c|c|c|c|c|c|c|c|c|}
\hline Number of lobes $n$ & $\mathrm{n}=1$ & $\mathrm{n}=2$ & $\mathrm{n}=3$ & $\mathrm{n}=4$ & $n=5$ & $n=6$ & $\mathrm{n}=7$ & $\mathrm{n}=8$ & $\mathrm{n}=9$ & $\mathrm{n}=10$ \\
\hline Axial frequency $\mathrm{f}_{1}, \mathrm{~Hz}$ & 219.38 & 340.84 & 476.85 & 618.26 & 762.14 & 907.78 & 1054.1 & 1200.9 & 1348.1 & 1495.5 \\
\hline $\begin{array}{c}\text { Circumferential frequency } \mathrm{f}_{2}, \\
\mathrm{~Hz}\end{array}$ & 113.10 & 187.50 & 270.32 & 356.07 & 442.89 & 529.98 & 617.39 & 704.96 & 792.5 & 880.14 \\
\hline Radial frequency $\mathrm{f}_{3}, \mathrm{~Hz}$ & 111.33 & 46.96 & 53.95 & 94.28 & 47.43 & 69.184 & 95.09 & 124.96 & 158.7 & 196.5 \\
\hline
\end{tabular}

Pressurized condition for different values of $1 / \mathrm{a}$ ratio

Table.9. $\mu=0.3,1 / \mathrm{a}=706 / 170=4.15, \lambda=0.756, \Delta \mathrm{P}=100 \mathrm{bar}$

\begin{tabular}{|c|c|c|c|c|c|c|c|c|c|c|}
\hline Number of lobes $\mathrm{n}$ & $\mathrm{n}=1$ & $\mathrm{n}=2$ & $\mathrm{n}=3$ & $\mathrm{n}=4$ & $\mathrm{n}=5$ & $\mathrm{n}=6$ & $\mathrm{n}=7$ & $\mathrm{n}=8$ & $\mathrm{n}=9$ & $\mathrm{n}=10$ \\
\hline Axial frequency $\mathrm{f}_{1}, \mathrm{~Hz}$ & 227.30 & 347.06 & 481.80 & 623.04 & 767.39 & 910.46 & 1056.4 & 1202.8 & 1350.0 & 1497.3 \\
\hline $\begin{array}{c}\text { Circumferential frequency } \mathrm{f}_{2}, \\
\mathrm{~Hz}\end{array}$ & 125.02 & 196.33 & 273.70 & 359.84 & 447.70 & 531.82 & 618.9 & 706.45 & 793.81 & 881.42 \\
\hline${\text { Radial frequency } \mathrm{f}_{3}, \mathrm{~Hz}}^{\mathrm{H}}$ & 54.32 & 92.643 & 93.42 & 123.11 & 156.892 & 72.750 & 98.62 & 127.93 & 163.35 & 207.19 \\
\hline
\end{tabular}

Table.10. $\mu=0.3,1 / \mathrm{a}=906 / 170=5.329, \lambda=0.589, \lambda=0.589, \Delta \mathrm{P}=100 \mathrm{bar}$

\begin{tabular}{|c|c|c|c|c|c|c|c|c|c|c|}
\hline Number of lobes $n$ & $\mathrm{n}=1$ & $\mathrm{n}=2$ & $\mathrm{n}=3$ & $\mathrm{n}=4$ & $\mathrm{n}=5$ & $\mathrm{n}=6$ & $\mathrm{n}=7$ & $\mathrm{n}=8$ & $\mathrm{n}=9$ & $\mathrm{n}=10$ \\
\hline Axial frequency $\mathrm{f}_{1}, \mathrm{~Hz}$ & 219.58 & 341.04 & 476.99 & 618.37 & 762.38 & 907.84 & 1054.1 & 1201.0 & 1345.8 & 1495.6 \\
\hline $\begin{array}{c}\text { Circumferential frequency } \mathrm{f}_{2}, \\
\mathrm{~Hz}\end{array}$ & 114.16 & 188.33 & 342.84 & 356.56 & 443.24 & 530.35 & 617.71 & 705.21 & 804.48 & 880.4 \\
\hline Radial frequency $\mathrm{f}_{3}, \mathrm{~Hz}$ & 10.44 & 68.54 & 106.96 & 51.23 & 72.45 & 95.44 & 122.65 & 153.48 & 152.04 & 226.36 \\
\hline
\end{tabular}

Table.11. $\mu=0.3,1 / \mathrm{a}=706 / 170=4.15, \lambda=0.756, \lambda=0.756, \Delta \mathrm{P}=200 \mathrm{bar}$

\begin{tabular}{|c|c|c|c|c|c|c|c|c|c|c|}
\hline Number of lobes $n$ & $\mathrm{n}=1$ & $\mathrm{n}=2$ & $\mathrm{n}=3$ & $\mathrm{n}=4$ & $\mathrm{n}=5$ & $\mathrm{n}=6$ & $\mathrm{n}=7$ & $\mathrm{n}=8$ & $\mathrm{n}=9$ & $\mathrm{n}=10$ \\
\hline Axial frequency $\mathrm{f}_{1}, \mathrm{~Hz}$ & 228.62 & 348.93 & 482.70 & 624.88 & 768.99 & 910.52 & 1056.4 & 1202.8 & 1351.9 & 1497.63 \\
\hline $\begin{array}{c}\text { Circumferential frequency } \mathrm{f}_{2}, \\
\mathrm{~Hz}\end{array}$ & 126.44 & 197.21 & 274.65 & 360.92 & 448.81 & 531.91 & 618.98 & 706.45 & 793.96 & 881.80 \\
\hline Radial frequency $\mathrm{f}_{3}, \mathrm{~Hz}$ & 54.99 & 94.006 & 95.903 & 128.81 & 59.741 & 75.583 & 110.60 & 141.28 & 173.44 & 215.31 \\
\hline
\end{tabular}

Table.12. $\mu=0.3,1 / \mathrm{a}=906 / 170=5.329, \lambda=0.589, \Delta \mathrm{P}=200 \mathrm{bar}$

\begin{tabular}{|c|c|c|c|c|c|c|c|c|c|c|}
\hline Number of lobes $n$ & $\mathrm{n}=1$ & $\mathrm{n}=2$ & $\mathrm{n}=3$ & $\mathrm{n}=4$ & $\mathrm{n}=5$ & $\mathrm{n}=6$ & $\mathrm{n}=7$ & $\mathrm{n}=8$ & $\mathrm{n}=9$ & $\mathrm{n}=10$ \\
\hline Axial frequency $\mathrm{f}_{1}, \mathrm{~Hz}$ & 219.83 & 341.26 & 479.13 & 511.23 & 762.50 & 907.91 & 1054.2 & 1201.1 & 1348.3 & 1484.7 \\
\hline $\begin{array}{c}\text { Circumferential frequency } \mathrm{f}_{2}, \\
\mathrm{~Hz}\end{array}$ & 115.11 & 189.20 & 271.66 & 358.16 & 443.66 & 530.77 & 618.05 & 705.49 & 793.07 & 930.80 \\
\hline Radial frequency $\mathrm{f}_{3}, \mathrm{~Hz}$ & 34.64 & 84.66 & 89.962 & 112.98 & 89.60 & 115.71 & 145.06 & 177.41 & 213.26 & 76.80 \\
\hline
\end{tabular}


Table13. Theoretical calculation of Eigen vectors at different Pressures

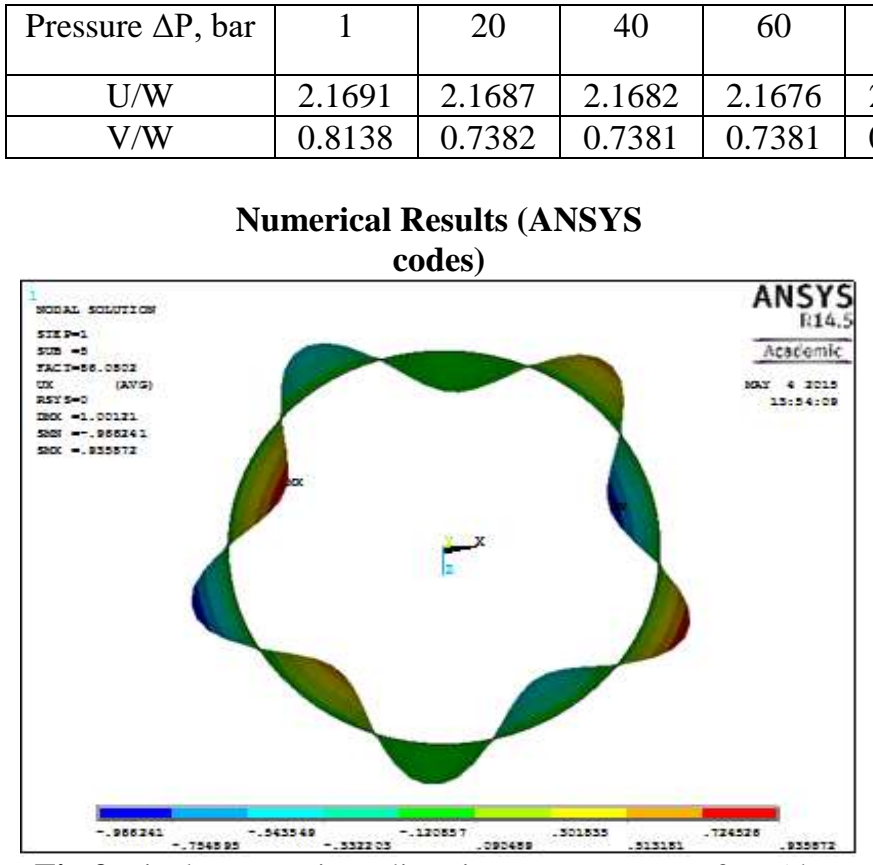

Fig.3 Displacement in $\mathrm{x}$-direction at a pressure of $\mathrm{P}=5$ bar

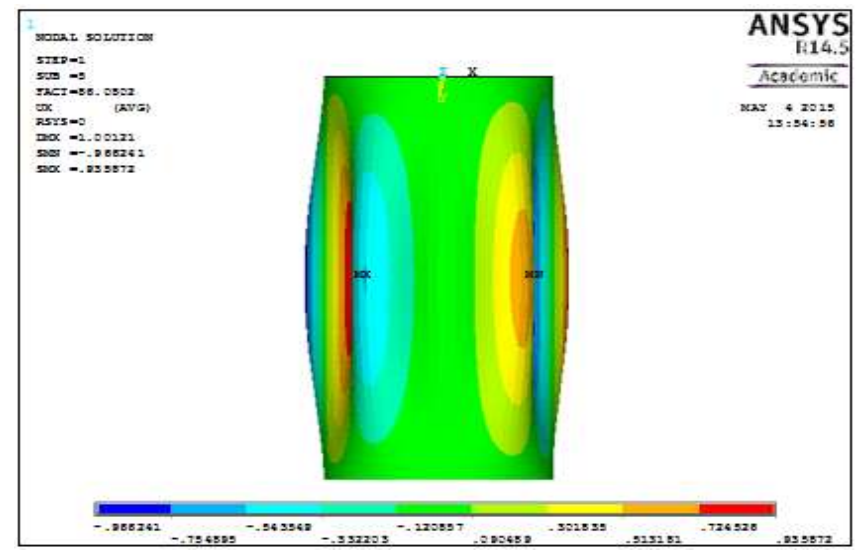

Fig.4 Displacement in $\mathrm{x}$-direction at a pressure of $\mathrm{P}=5$ bar

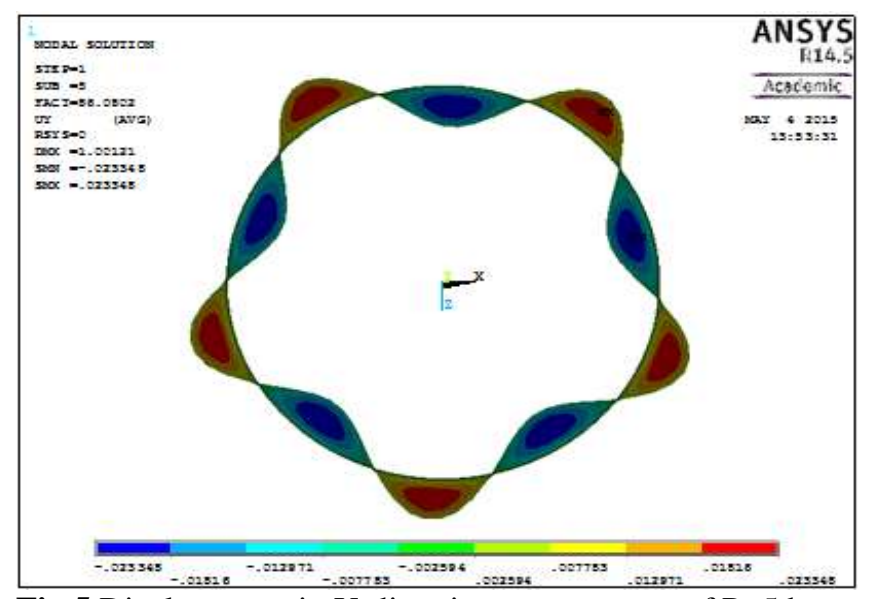

Fig.5 Displacement in Y-direction at a pressure of $\mathrm{P}=5$ bar

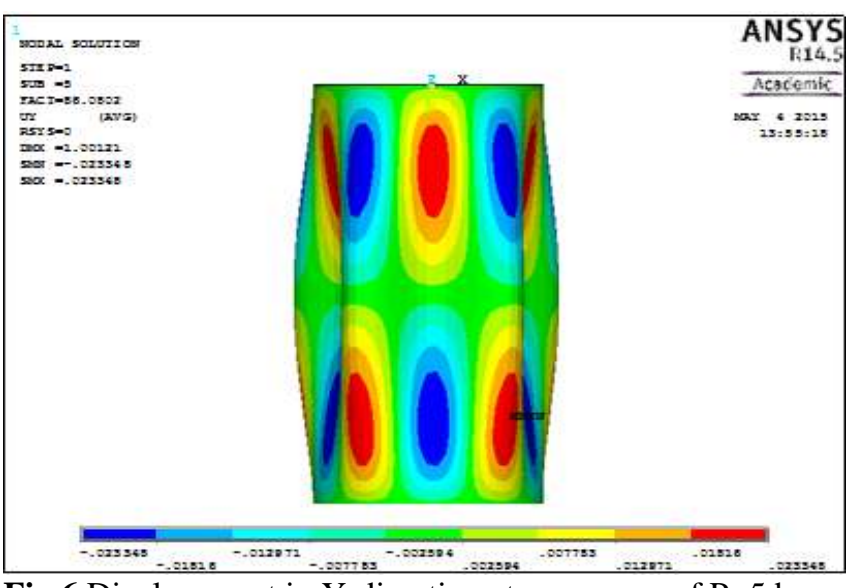

Fig.6 Displacement in Y-direction at a pressure of $\mathrm{P}=5$ bar

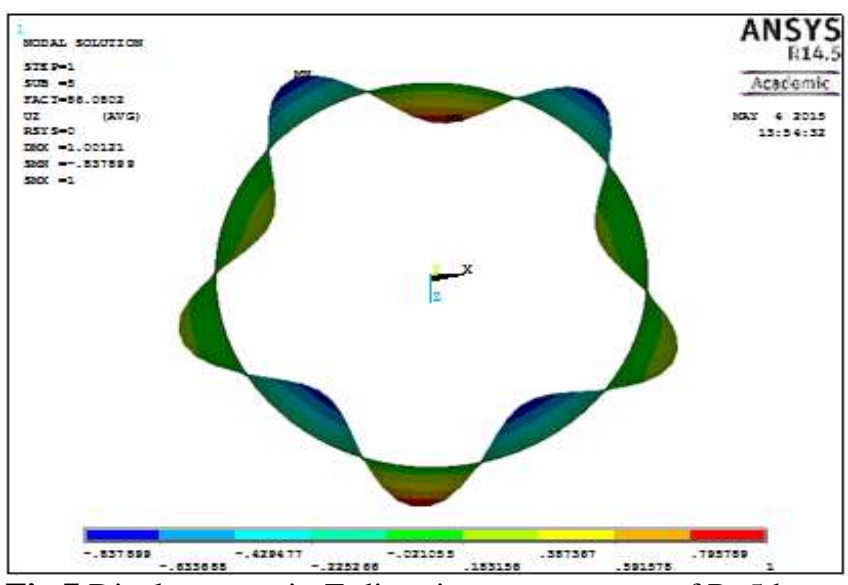

Fig.7 Displacement in Z-direction at a pressure of $\mathrm{P}=5$ bar

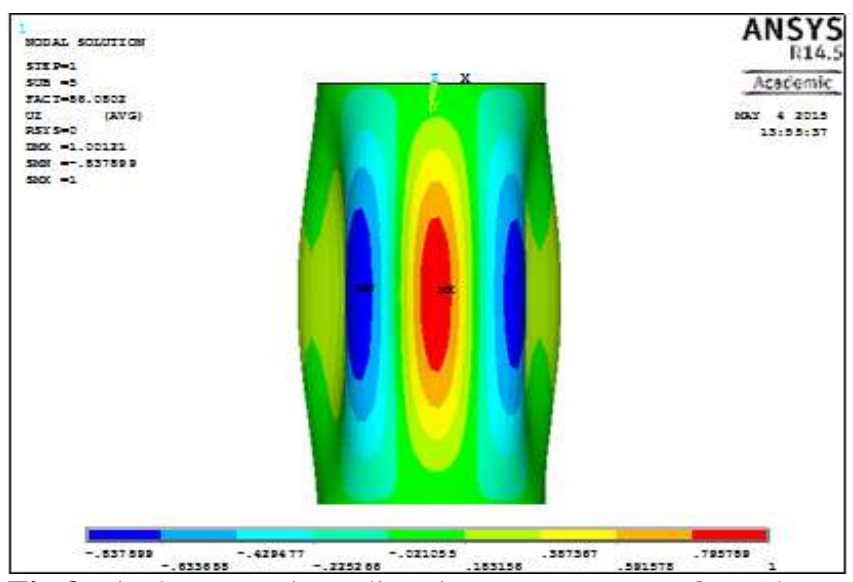

Fig.8 Displacement in Z-direction at a pressure of $\mathrm{P}=5$ bar

Comparison of ANSYS results with the theoretical results,

For Pressure $\mathrm{P}=5$ bar and at nodal value $\mathrm{n}=5$ 
Table.14. Validation of Eigen vectors with numerical technique

\begin{tabular}{|l|l|l|l|}
\hline \multicolumn{2}{|l|}{ Theoretical results } & \multicolumn{2}{l|}{ ANSYS results } \\
\hline $\mathrm{U} / \mathrm{W}$ & V/W & $\mathrm{U} / \mathrm{W}$ & V/W \\
\hline 0.2480 & 2.408 & 0.223 & 2.438 \\
\hline
\end{tabular}

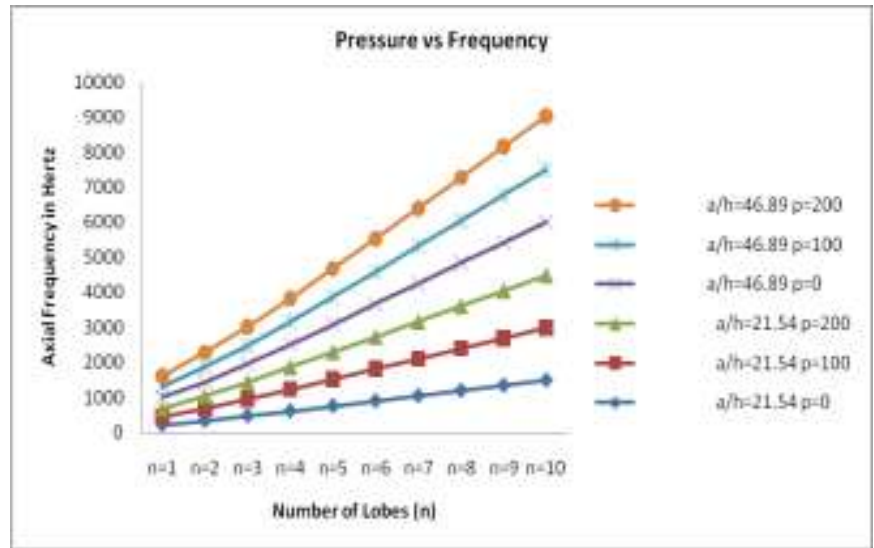

Fig.9 Frequency variation in axial direction for varying pressure values and $\mathrm{a} / \mathrm{h}$ ratios.

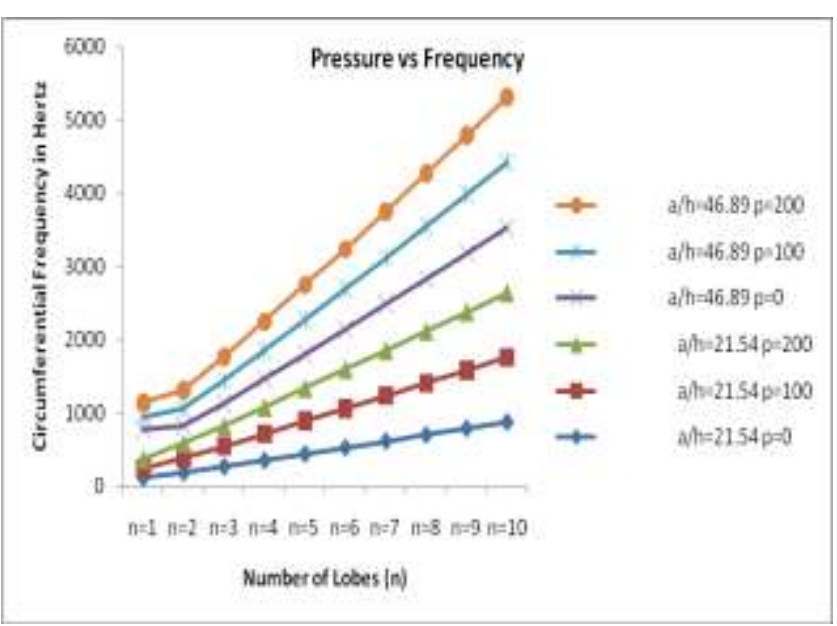

Fig.10. Frequency variation in circumferential direction for varying pressure values and $\mathrm{a} / \mathrm{h}$ ratios.

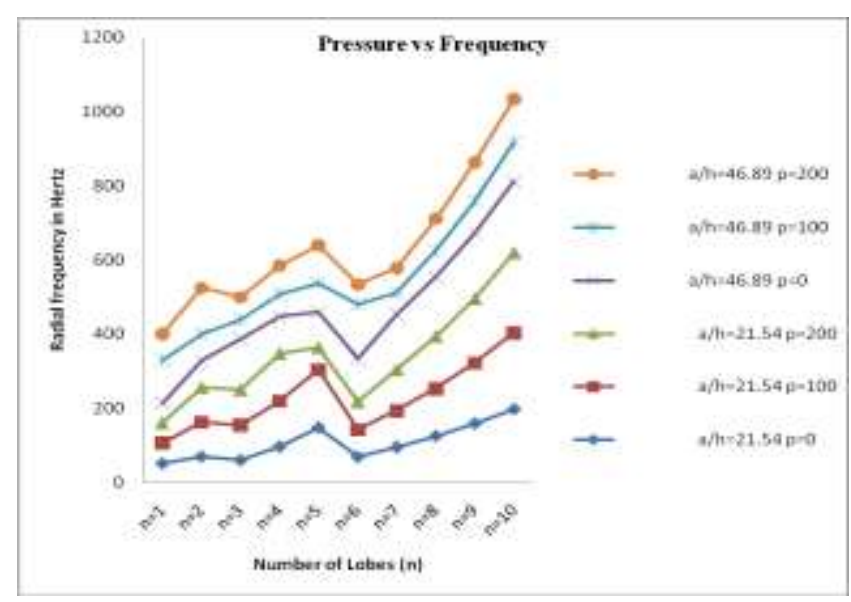

Fig.11. Frequency variation in radial direction for varying pressure values and $\mathrm{a} / \mathrm{h}$ ratios.

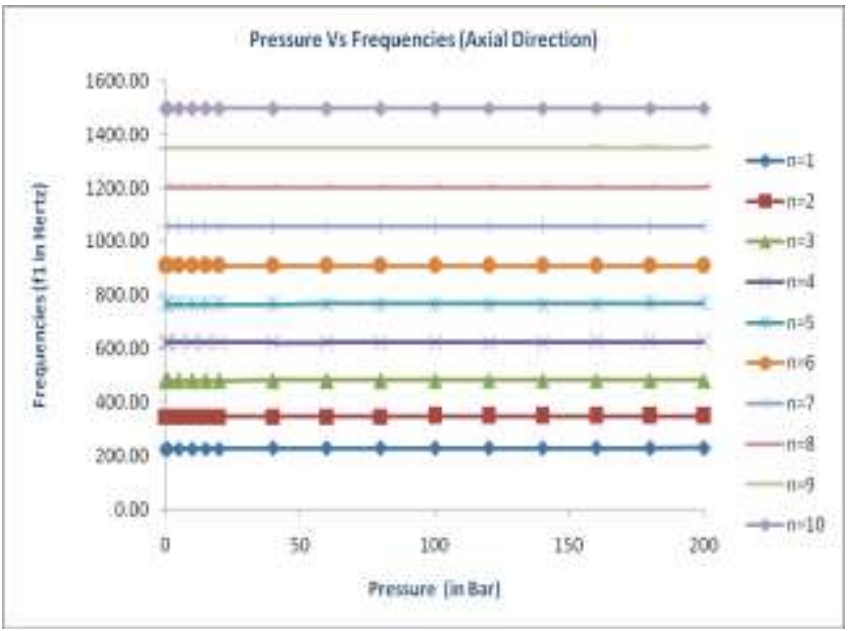

Fig.12 Frequency variation in axial direction for different pressure values, with varying number of lobes.

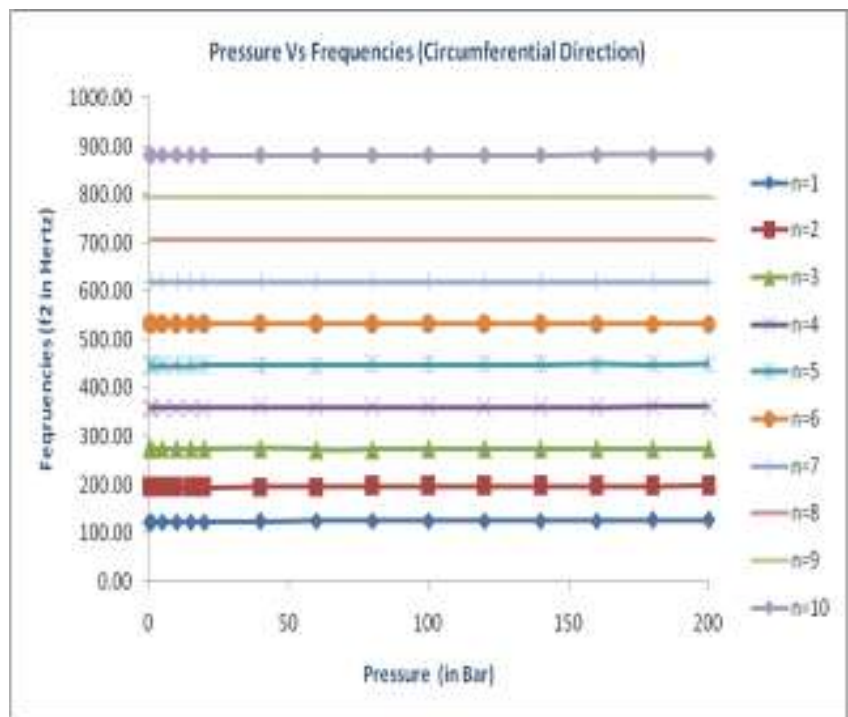

Fig.13. Frequency variation in circumferential direction for different pressure values, with varying number of lobes.

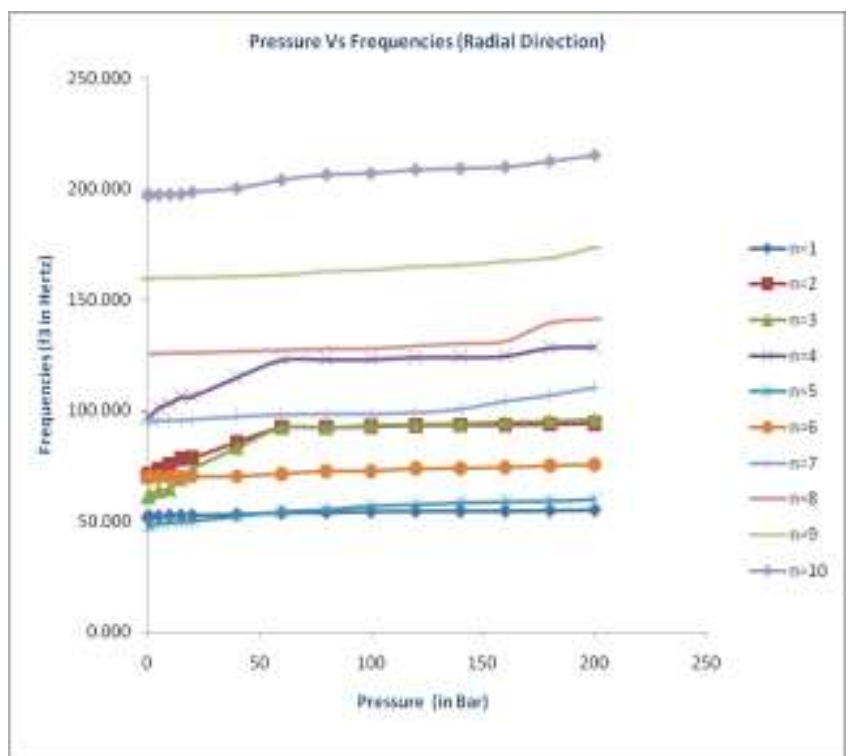

Fig.14. Frequency variation in radial direction for different pressure values, with varying number of lobes. 


\section{RESULTS AND DISCUSSION}

The results are developed under two categories viz., Pressurized and Unpressurised conditions.

\section{UNPRESSURIZED CONDITIONS}

By considering the Love's assumptions the governing equations of thin cylindrical shells is determined and by applying certain assumptions to the higher order linear differential equations, characteristic equation is formulated. The characteristic equation is a cubic equation with three constants $\mathrm{K}_{0}, \mathrm{~K}_{1}$ and $\mathrm{K}_{2}$, that computes the frequency values in axial, circumferential and radial direction. Under unpressurized condition, the natural frequency values in axial, circumferential and radial direction are observed to increase at lower rates with the increase in the circumferential nodal value ' $n$ '. The percentage increase in frequency values gradually decreases when it reaches high values of ' $n$ ' (i.e. from $30 \%$ to $15 \%$ ). The minimum and maximum frequency values computed in axial direction $n=1$ is $226.45 \mathrm{~Hz}$ and increases to a value of $1497.2 \mathrm{~Hz}$ at $\mathrm{n}=10$. In circumferential direction the frequency value at $n=1$ is $121.83 \mathrm{~Hz}$ and increases to a value of $881.28 \mathrm{~Hz}$ at $\mathrm{n}=10$. In radial direction the frequency value at $\mathrm{n}=1$ is $52.08 \mathrm{~Hz}$ and increases to a maximum value of $197.03 \mathrm{~Hz}$ at $\mathrm{n}=10$.

\section{PRESSURIZED CONDITIONS}

By considering the LOVE'S assumptions the governing equations of thin cylindrical shells is determined and by applying certain assumptions to the higher order linear differential equations and by replacing the membrane stresses as a function pressure the characteristic equation is determined. The characteristic equation obtained is a cubic equation with ten constants $\mathrm{K}_{0}, \mathrm{~K}_{1}, \mathrm{~K}_{2}, \mathrm{a}_{1}, \mathrm{a}_{2}, \mathrm{a}_{3}, \mathrm{a}_{4}, \mathrm{a}_{5}, \mathrm{~b}_{1}$ and $b_{2}$. Under pressurized condition frequency behavior is analyzed at different values of pressure ranging from a minimum value of 1 bar to a maximum value 200 bar. It is observed that with the increase in the pressure value has a negligible effect in the natural frequency values in axial, circumferential and radial direction. In axial direction the frequency value at $\mathrm{P}=1$ bar for $\mathrm{n}=2$ is $346.62 \mathrm{~Hz}$ and at the maximum pressure of $\mathrm{P}=200 \mathrm{bar}$ for $\mathrm{n}=2$ frequency is 348.93 Hz. In circumferential direction the frequency value at $\mathrm{P}=1$ bar for $\mathrm{n}=2$ is $193.92 \mathrm{~Hz}$ and at the maximum pressure of $\mathrm{P}=200$ bar for $\mathrm{n}=2$ frequency is $197.21 \mathrm{~Hz}$, which shows that the variation of natural frequency is too small in both the directions. It is also noted that with the increase in the pressure value the natural frequency values in radial direction significantly increases at higher rates, i.e. in radial direction the frequency value at $\mathrm{P}=1$ bar for $\mathrm{n}=2$ is $70.29 \mathrm{~Hz}$ and at the maximum pressure of $\mathrm{P}=200$ bar for $\mathrm{n}=2$ frequency is $94.006 \mathrm{~Hz}$. Simulations for cylindrical shell subjected to uniform internal pressure of 5bar is carried out to compute Eigen vectors and Eigen values for all three directions of the shell. It is observed that findings of the theoretical model are very close to the results computed through the numerical technique, ANSYS.

\section{CONCLUSIONS}

The larger the number of circumferential waves ' $n$ ' the faster is the rate of increase of frequency with internal pressure. The significant increase in the lowest frequency with increase in pressure is caused by the fact that ' $n$ ' is fairly large at the lowest frequency if the cylinder is short and if the wall is very thin typically $\mathrm{a} / \mathrm{h}=21.54$. The frequency values gradually increases with the increase in the circumferential nodes $\mathrm{n}$ at a rate of $0.2 \%$ to $0.3 \%$. The variation of Eigen value, $\varphi$ with pressure value is small for small ' $n$ '. At higher values of ' $n$ ' the variation is considerably higher than that of the lower nodes. The axial and the circumferential frequencies increases in small amounts as ' $n$ ' value increases, where as the frequency in radial direction rapidly increases with the increase in the pressure values. The influence of physical parameter, $(\mathrm{a} / \mathrm{h})$ (radius to thickness ratio) is prominent and observed to have rapid increase in frequencies at lower nodal values rather than at higher nodes. The physical parameter, (1/a) (length to radius ratio) does not have significant effect on frequencies with increase in nodes. The frequency variation in axial and circumferential directions of thin shell is constant for varied internal pressures. However the frequency variation in radial direction of thin shell is observed to be varying mostly at lower nodes.

\section{REFERENCES}

[1]. Love, A. E. H., (1927) Mathematical Theory of Elasticity. $4^{\text {th }}$ Edition, Cambridge;34-38

[2]. J. Ramachandran, (1993) Thin shells, Theory and problems. University Press (India) Ltd.: 267 - 268

[3]. Timoshenko. S. (1940) Theory of Plates and Shells, McGraw-Hill: 100-104

[4]. Arnold. R. N, Warburton, G. B. (1949) Flexural Vibrations of the Walls of Thin Cylindrical Shells having Freely Supported Ends, Proceedings of Royal Society. A, 197.:238 - 244.

[5]. Fllugge W (1934) Static and Dynamic Analysis, Schalen. Juliug Springer, e Berlin: 35-40.

[6]. Reissner, E (1955) Non-linear Effects in Vibrations of Cylindrical Shells, Ramo-Wooldridge Report: 30 - 33.

[7]. Reissner, E (1955) On Transverse Vibrations of Thin Shallow Elastic Shells. Quarterly of Applied Maths, :169-176.

[8]. Nitin S Gokhale (2008) Practical Finite Element Analysis, Finite to Infinite Publishers;100-106

\section{NOMENCLATURE}

$\begin{array}{lc}a_{1}, a_{2}, b_{1}, b_{2} & \text { etc coefficients in } K^{s}{ }_{0}, K^{v}{ }_{1}, K^{s}{ }_{2} . \\ \mathrm{E} & \text { young's modulus } \\ \mathrm{f}=\frac{\omega}{2 \pi} & \text { frequency, cycles per sec } \\ \mathrm{h} & \text { thickness of the wall } \\ K_{0}, K_{1}, K_{2}, K^{s}{ }_{0} & \text { etc coefficients in frequency equation } \\ \mathrm{L} & \text { length of cylinder } \\ \mathrm{m} & \text { number of axial half -waves } \\ \mathrm{n} & \text { number of circumferential waves }\end{array}$


$N_{X}, N_{\theta}, N_{X \theta}, Q_{x}, Q_{y} \quad$ stress resultants in shell, force per unit length

$\overline{N_{x}}, \overline{N_{\theta}} \quad$ membrane stresses due to internal pressure

$$
\overline{n_{\theta}}=\frac{\overline{N_{\theta}}}{E h} \quad n_{\theta}=\left(1-\vartheta^{2}\right)
$$

membrane stresses due to internal pressure

$$
\begin{array}{lc}
\overline{n_{x}}=\frac{\overline{N_{x}}}{E h} & n_{x}=\left(1-\vartheta^{2}\right) \overline{n_{x}} \\
k=\frac{h}{a} \quad \text { thickness parameter } \\
\beta=\frac{h^{2}}{12 a^{2}} \quad \text { shell parameter } \\
\delta=\left(1-\vartheta^{2}\right) x \quad \text { frequency parameter } \\
\lambda=\frac{\pi m a}{L} \quad \text { axial wave length factor } \\
\vartheta \quad \text { Poisson's ratio }
\end{array}
$$

